\title{
PRESENÇA DE SÍNDROME METABÓLICA EM PACIENTES EXPOSTOS A ANTIPSICÓTICOS: UMA REVISÃO SISTEMÁTICA
}

\author{
Rita de Kássia Alves de Oliveira1, Bianca Norrara Costa Gomes da Silva², \\ Francisca Overlânia Vieira Lima², Marco Aurélio de Moura Freire ${ }^{2}$ \\ ${ }^{1}$ Nutricionista Residente, Programa de Pós-Graduação na modalidade de Residência \\ Multiprofissional em Atenção Básica/ Saúde da Família e Comunidade, Universidade do Estado \\ do Rio Grande do Norte, Mossoró-RN, Brasil. \\ ${ }^{2}$ Curso de Pós-Graduação em Saúde e Sociedade (PPGSS), Faculdade de Ciências da Saúde, \\ Universidade do Estado do Rio Grande do Norte, Mossoró, RN, Brasil. \\ E-mail para correspondência: kassia alvs@hotmail.com
}

\begin{abstract}
Resumo
A administração de fármacos antipsicóticos representa uma intervenção amplamente utilizada na prática clínica como parte do tratamento de pessoas com transtorno psíquico. Contudo, a literatura tem associado o uso dessa classe de medicamentos a alterações metabólicas, caso não administrados de maneira correta. Objetivou-se avaliar a presença da Síndrome Metabólica (SM) e sua relação com a utilização de antipsicóticos. Revisão sistemática realizada de abril a maio de 2021, nas bases de dados: MedLine, LILACS, Portal de Periódicos CAPES e SCIELO, utilizando os seguintes descritores: Antipsicóticos, Síndrome Metabólica, Perfil Nutricional, junto aos critérios de inclusão e exclusão. Verificouse relação bem documentada do uso de antipsicóticos atípicos e alterações metabólicas em pacientes diagnosticados com esquizofrenia. Aponta-se como limitações dos estudos a dificuldade em identificar a Síndrome Metabólica devido à ausência de um padrão-ouro decorrente das distintas classificações existentes, bem como ausência de dados quanto ao consumo alimentar e estilo de vida dos pacientes, que permitiriam o estabelecimento de uma melhor associação entre os antipsicóticos e Síndrome Metabólica.
\end{abstract}

Palavras-chave: antipsicóticos, síndrome metabólica, perfil nutricional.

\begin{abstract}
The administration of antipsychotic drugs represents an intervention widely used in clinical practice as part of the treatment of people with mental disorders. However, the literature has associated the use of this class of drugs with metabolic changes, if not properly administered. The objective was to evaluate the presence of Metabolic Syndrome (MS) and its relationship with the use of antipsychotics. Systematic review carried out from April to May 2021, in the databases: MedLine, LILACS, CAPES and SCIELO Journals Portal, using the following descriptors: Antipsychotics, Metabolic Syndrome, Nutritional Profile, along with the inclusion and exclusion criteria. There was a well-documented relationship between the use
\end{abstract}


of atypical antipsychotics and metabolic changes in patients diagnosed with schizophrenia. As limitations of the studies, it is pointed out the difficulty in identifying the Metabolic Syndrome due to the absence of a gold standard resulting from the different existing classifications, as well as the absence of data regarding the patients' food consumption and lifestyle, which would allow the establishment of a better association between antipsychotics and Metabolic Syndrome.

Keywords: antipsychotics, metabolic syndrome, nutritional profile.

\section{Introdução}

As questões tocantes à saúde psíquica têm sido pauta de grandes discussões e transformações na maneira de assistir a parcela da população acometida por transtornos mentais. Nesse contexto, a farmacoterapia tem representado uma das principais medidas de intervenção adotada no tratamento destes pacientes (XAVIER et al., 2014).

Rocha et al. (2013) afirmam que o emprego dos fármacos antipsicóticos tem sido cada vez mais frequente na prática clínica, podendo-se atribuir tal ocorrência a vários fatores, dentre os quais a evolução e descobertas da ciência relativa à saúde mental, possibilitando uma maior ocorrência de diagnósticos de transtornos psíquicos na população, inserção de novos fármacos no mercado farmacêutico e novas indicações terapêuticas dos medicamentos já existentes, bem como a quebra do estigma quanto às doenças psicológicas.

O tratamento medicamentoso visa reduzir os sintomas prejudiciais das patologias psiquiátricas, com propósito de melhor adaptação à realidade. Por outro lado, apresenta vários efeitos secundários, podendo-se citar alteração no estado nutricional e risco metabólico em pacientes que utilizam substâncias psicoativas (XAVIER et al., 2014).

Zortéa et al. (2010) apontam que a conduta terapêutica para pessoas com transtornos mentais focaliza na remissão dos sintomas psicóticos, negligenciando, por vezes, outros aspectos importantes, como a segurança cardiovascular, distúrbios metabólicos e outros sintomas depreciativos, o que resulta em uma maior prevalência de morbidade clínica e agravo na qualidade de vida dos pacientes que utilizam psicofármacos. Porém, quando bem administrados, os psicotrópicos tornam-se grandes aliados no processo de cuidado do usuário. 
Desse modo, é de fundamental relevância que seja dada maior atenção aos efeitos dos antipsicóticos sob os riscos metabólicos destes indivíduos, visto que na prática clínica os efeitos secundários dos medicamentos em questão são negligenciados, por muitas vezes, pelo desaparecimento dos sintomas psíquicos.

O que motivou a realização do presente estudo foi à relevância de oferecer um levantamento científico atual sobre os impactos dos antipsicóticos no perfil metabólico de usuários dessa classe farmacológica, de modo a possibilitar uma melhor compreensão e clareza dessa associação na prática clínica, buscando colaborar para um tratamento mais consciente que garanta não apenas a minimização dos sintomas psíquicos, como também ofereça uma melhor qualidade de vida aos usuários destes medicamentos.

Considera-se para este artigo que a revisão sistemática se preocupou em buscar novas evidências que permitam compreender quais possíveis fatores e condições ocasionam o desenvolvimento da síndrome metabólica em usuários expostos a antipsicóticos, visando possibilitar uma melhor compreensão acerca da temática. Neste sentido, objetivou-se avaliar a presença da Síndrome Metabólica (SM) e sua relação com a utilização de antipsicóticos.

\section{Metodologia}

O presente estudo corresponde a uma revisão sistemática da literatura. Para tanto, recorreu-se aos sistemas de busca eletrônica de artigos científicos indexados nas bases de dados Medical Literature Analyses and Retrieval System Online (MedLine), Literatura LatinoAmericana e do Caribe em Ciências da Saúde (LILACS), Portal de Periódicos CAPES e Scientific Electronic Library Online (SCIELO). O estudo foi norteado por produções cientificas publicadas no período compreendido entre os anos de 2014 a 2019 sobre a presença da Síndrome Metabólica e sua relação com a utilização de antipsicóticos.

A amostra compreendeu artigos indexados publicados em periódicos que seguiu os seguintes critérios de inclusão: a) Veículo de publicação: optou-se por periódicos indexados, visto que divulgam mais os artigos e são de fácil acesso; b) Idioma de publicação: artigos publicados na íntegra em língua inglesa, portuguesa e espanhola; c) Ano de publicação: selecionaram-se artigos do período de 2014 a 2019; d) Referências que tratassem dos 
descritores: Antipsicóticos, Síndrome Metabólica e Perfil Nutricional. Sendo utilizado o cruzamento Antipsicóticos x Síndrome Metabólica e Antipsicótico x Perfil Nutricional. O quadro 1 expõe uma síntese da seleção de artigos por descritores.

Quadro 1. Síntese dos resultados da busca de artigos por descritores

\begin{tabular}{|l|c|c|c|}
\hline \multicolumn{1}{|c|}{ Descritores } & $\begin{array}{c}\text { Textos } \\
\text { encontrados }\end{array}$ & $\begin{array}{c}\text { Textos } \\
\text { na } \\
\text { íntegra }\end{array}$ & $\begin{array}{c}\text { Textos com } \\
\text { exigências } \\
\text { requeridas }\end{array}$ \\
\hline $\begin{array}{l}\text { Antipsicóticos /Síndrome } \\
\text { Metabólica }\end{array}$ & 129 & 117 & 12 \\
\hline Antipsicótico/Perfil Nutricional & 1 & 1 & 1 \\
\hline Total & 130 & 118 & 13 \\
\hline
\end{tabular}

Fonte: Dados da pesquisa (2020)

Foram excluídos da Revisão Sistemática, dissertações, trabalhos de conclusão de curso e teses, artigos que não corresponderam a temática proposta, pesquisas publicadas precedentes à 2014, estudos que utilizaram como público-alvo crianças e adolescentes.

O processo de localização e seleção dos artigos ocorreu em três etapas. A primeira etapa consistiu na leitura dos títulos dos artigos, que foram localizados nas buscas, a fim de excluir aqueles que claramente não atendiam aos critérios de inclusão previamente estabelecidos. Na segunda etapa foi realizada a leitura dos resumos dos artigos, e quando o resumo não continha informações suficientes para tomada de decisão quanto à inclusão ou exclusão, o artigo era mantido para a próxima etapa do processo de revisão. A terceira e última etapa incluiu a análise dos artigos na íntegra. Com as produções, foi feita uma leitura do artigo na íntegra de cada pesquisa, identificando as ideias centrais, a divisão dos principais achados e a síntese dos resultados.

De modo a melhorar a organização e compreensão do material encontrado, os dados foram devidamente tabulados, sendo os resultados analisados seguindo uma categorização, por meio de 11 variáveis, quais sejam: título, autores, revista/ano de publicação, base de dados utilizada, objetivos, participantes (critérios para seleção da amostra; tipo de amostra), 
intervenção (procedimentos utilizados), local onde a pesquisa foi realizada (cidade/estado/país/local), resultados obtidos, limites/dificuldades e conclusões/considerações finais. A partir disso, foi realizada a análise dos conteúdos dos artigos, com o intuito de obter um cenário da produção nacional e internacional no tocante a influência dos antipsicóticos no desencadeamento dos fatores de risco para Síndrome Metabólica.

Dessa maneira, por meio da adoção dos rigorosos critérios supracitados, os artigos que exploram a influência dos antipsicóticos na indução da Síndrome Metabólica, indexados nas 4 bases de dados utilizadas, acredita-se que os estudos selecionados sejam os atuais mais relevantes sobre o tema pesquisado.

\section{Resultados e discussão}

Foi selecionado um total de 129 artigos no primeiro rastreamento nas bases de dados. Foi obtido um total de 117 estudos, sendo um excluído devido duplicidade e dois por não atenderem aos critérios necessários. Posteriormente, 93 artigos foram excluídos após leitura do título e do resumo. Dos 24 artigos restantes, 11 foram excluídos após leitura de seu conteúdo de modo integral, por não estarem diretamente associados ao tema do estudo. Assim, 13 produções atenderam as exigências requeridas. A Figura 1 mostra um resumo do processo de seleção dos artigos revisados.

Os 13 artigos analisados foram publicados nos últimos seis anos. Destes, 12 artigos, dois são revisões de literatura, um é uma meta-análise. Quanto ao tipo de estudo, oito são estudos de cunho transversal, um corresponde a estudo prospectivo longitudinal e um a estudo metabolômico. Aponta-se, ainda, que houve uma prevalência de estudos $(n=9)$ de caráter internacional, observando-se uma maior concentração nos países Asiáticos $(n=4)$, sendo quatro artigos de âmbito nacional. As características dos estudos (ano de publicação, periódico, local de realização e tipo de estudo) estão sintetizadas no quadro 2.

No quadro 3, observa-se que o diagnóstico para o transtorno psiquiátrico de esquizofrenia, foi referido nos 13 artigos incluídos no estudo. Dentre os mesmos artigos analisados, foram verificados concomitantemente, em menor 
proporção, diagnósticos de Transtorno depressivo maior e Transtorno do humor bipolar.

De acordo com Paredes et al. (2014), Seow et al. (2017) e Silva et al. (2019), a esquizofrenia é uma doença psiquiátrica grave caracterizada pela presença de sintomas positivos (alucinações, delírios, discurso desorganizado, comportamento psicomotor anormal), e sintomas negativos, caracterizados pela perda de funções comportamentais consideradas normais (tais como apatia e retraimento social). O início da esquizofrenia geralmente ocorre durante a fase adulta, com várias consequências socioeconômicas.

Figura 1: Fluxograma do processo de seleção de estudos

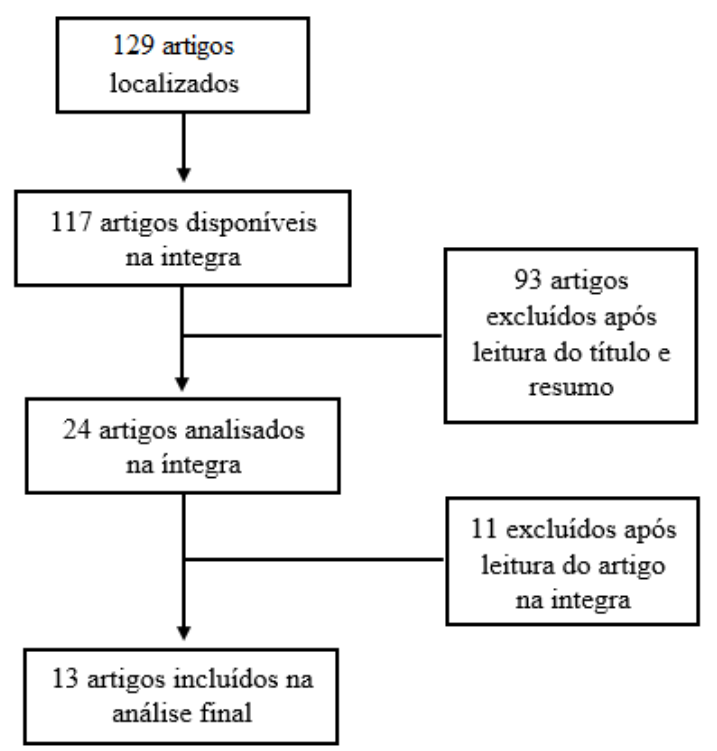

Fonte: Dados da pesquisa (2020) 
Quadro 2. Principais características dos artigos selecionados

\begin{tabular}{|c|c|c|c|c|}
\hline Autor & Periódico & Local & $\begin{array}{l}\text { Tipo de } \\
\text { estudo }\end{array}$ & Objetivo \\
\hline $\begin{array}{l}\text { Seow et } \\
\text { al., } 2017\end{array}$ & $\begin{array}{l}\text { Comprehensiv } \\
\text { e Psychiatry }\end{array}$ & $\begin{array}{c}\text { Singapur } \\
\mathrm{a}\end{array}$ & Meta-análise & $\begin{array}{l}\text { Determinar o } \\
\text { perfil } \\
\text { cardiometablico e } \\
\text { os fatores de } \\
\text { risco associados } \\
\text { em um grupo de } \\
\text { pacientes } \\
\text { institucionalizado } \\
\text { s com } \\
\text { esquizofrenia ou } \\
\text { a perturbação } \\
\text { esquizoafetiva em } \\
\text { cuidados } \\
\text { hospitalares na } \\
\text { instituição } \\
\text { psiquiátrica única } \\
\text { terciária } \\
\text { Singapura. em }\end{array}$ \\
\hline $\begin{array}{l}\text { Jeon et } \\
\text { al., } 2017\end{array}$ & $\begin{array}{c}\text { International } \\
\text { Journal of } \\
\text { Molecular } \\
\text { Sciences }\end{array}$ & $\begin{array}{l}\text { Coreia do } \\
\text { Sul }\end{array}$ & Revisão & $\begin{array}{lr}\text { Analisar } & \text { a } \\
\text { utilização } & \text { de } \\
\text { antipsicóticos } & \\
\text { atípicos } & \text { na } \\
\text { esquizofrenia } & \text { e } \\
\text { sua relação com } \\
\text { a síndrome } \\
\text { metabólica. }\end{array}$ \\
\hline $\begin{array}{l}\text { Alswat et } \\
\text { al., } 2017\end{array}$ & $\begin{array}{l}\text { Medical } \\
\text { Archives }\end{array}$ & $\begin{array}{l}\text { Arábia } \\
\text { Saudita }\end{array}$ & Transversal & $\begin{array}{lr}\text { Avaliar } & \text { a } \\
\text { prevalência } & \text { de } \\
\text { Síndrome } & \\
\text { Metabólica e } & \text { sua } \\
\text { relação com } & \text { as } \\
\text { modalidades } & \text { de } \\
\text { tratamento } & \text { e } \\
\text { diagnóstico } & \\
\text { psiquiátrico. } & \end{array}$ \\
\hline $\begin{array}{l}\text { Paredes et } \\
\text { al., } 2014\end{array}$ & $\begin{array}{l}\text { International } \\
\text { Journal of } \\
\text { Neuropsychop } \\
\text { harmacology }\end{array}$ & $\begin{array}{l}\text { Texas, } \\
\text { EUA }\end{array}$ & Metabolômico & $\begin{array}{lr}\text { Compreender a } \\
\text { relação entre os } \\
\text { diferentes } \\
\text { antipsicóticos de } \\
\text { segunda geração } \\
\text { e marcadores } \\
\text { metabólicos na } \\
\text { síndrome } \\
\text { metabólica } \\
\text { associada } \\
\text { esquizofrenia. à }\end{array}$ \\
\hline $\begin{array}{l}\text { Saloojee } \\
\text { et al., }\end{array}$ & $\begin{array}{c}\text { BMC } \\
\text { Psychiatry }\end{array}$ & $\begin{array}{l}\text { África do } \\
\text { Sul }\end{array}$ & $\begin{array}{c}\text { Corte } \\
\text { Transversal }\end{array}$ & $\begin{array}{ll}\text { Documentar } & \text { a } \\
\text { extensão } & \text { da }\end{array}$ \\
\hline
\end{tabular}




\begin{tabular}{|c|c|c|c|c|}
\hline 2014 & & & & $\begin{array}{l}\text { baixa frequência } \\
\text { esperada de } \\
\text { testes para todos } \\
\text { os componentes } \\
\text { da síndrome } \\
\text { metabólica em } \\
\text { pacientes com } \\
\text { SMl em um país } \\
\text { de baixa renda } \\
\text { média. }\end{array}$ \\
\hline $\begin{array}{r}\text { Silva et } \\
\text { al., } 2019\end{array}$ & $\begin{array}{c}\text { Brazilian } \\
\text { Journal of } \\
\text { Pharmaceutica } \\
\text { I Sciences }\end{array}$ & Brasil & Revisão & $\begin{array}{ll}\text { Compreender } & \text { os } \\
\text { efeitos } & \text { do } \\
\text { aripiprazol } & \text { na } \\
\text { Síndrome } & \\
\text { Metabólica } & \text { em } \\
\text { pacientes } & \\
\text { diagnosticados } & \\
\text { com } & \\
\text { esquizofrenia. } & \end{array}$ \\
\hline $\begin{array}{l}\text { Olmos et } \\
\text { al., } 2017\end{array}$ & $\begin{array}{c}\text { Revista } \\
\text { Psiquiatria de } \\
\text { Uruguay }\end{array}$ & Uruguai & $\begin{array}{l}\text { Prospectivo } \\
\text { longitudinal }\end{array}$ & $\begin{array}{l}\text { Determinar se } \\
\text { existe } \\
\text { correlação entre o } \\
\text { Síndrome } \\
\text { Metabólica e as } \\
\text { concentrações } \\
\text { plasmáticas de } \\
\text { clozapina em } \\
\text { uma população } \\
\text { de pacientes com } \\
\text { esquizofrenia do } \\
\text { Hospital } \\
\text { Vilardebó, } \\
\text { Uruguai. }\end{array}$ \\
\hline $\begin{array}{l}\text { Freitas et } \\
\text { al., } 2016\end{array}$ & $\begin{array}{c}\text { Ciencia y } \\
\text { Enfermería }\end{array}$ & $\begin{array}{c}\text { Oeste de } \\
\text { MG }\end{array}$ & $\begin{array}{l}\text { Descritivo e } \\
\text { transversal }\end{array}$ & $\begin{array}{l}\text { Estimar } \\
\text { prevalência de } \\
\text { síndrome } \\
\text { metabólica e seus } \\
\text { fatores } \\
\text { associados em } \\
\text { pacientes com } \\
\text { esquizofrenia } \\
\text { refratária em uso } \\
\text { do antipsicótico } \\
\text { clozapina. }\end{array}$ \\
\hline $\begin{array}{l}\text { Santini et } \\
\text { al., } 2016\end{array}$ & $\begin{array}{l}\text { Rivista di } \\
\text { Psichiatria }\end{array}$ & Itália & Transversal & $\begin{array}{l}\text { Estimar a } \\
\text { prevalência da } \\
\text { síndrome } \\
\text { metabólica em } \\
\text { uma amostra } \\
\text { psiquiátrica }\end{array}$ \\
\hline
\end{tabular}




\begin{tabular}{|c|c|c|c|c|}
\hline & & & & $\begin{array}{lr}\text { italiana, tratada } \\
\text { com diferentes } \\
\text { tipos r de } \\
\text { antipsicóticos. }\end{array}$ \\
\hline $\begin{array}{l}\text { Saloojee } \\
\text { et al., } \\
2016\end{array}$ & Plos One & África & Transversal & $\begin{array}{lr}\text { Determinar } & \text { a } \\
\text { prevalência e os } \\
\text { fatores de risco } \\
\text { para síndrome } \\
\text { metabólica } & \text { em } \\
\text { pacientes } & \text { sul- } \\
\text { africanos } & \text { com } \\
\text { doença } & \text { mental } \\
\text { grave. } & \end{array}$ \\
\hline $\begin{array}{l}\text { Sugawara } \\
\text { et al., } \\
2014\end{array}$ & Plos One & Japão & Transversal & $\begin{array}{lr}\text { Avaliar } & \text { as } \\
\text { atitudes } & \text { dos } \\
\text { psiquiatras em } \\
\text { relação a eventos } \\
\text { adversos } \\
\text { metabólicos em } \\
\text { pacientes com } \\
\text { esquizofrenia }\end{array}$ \\
\hline $\begin{array}{l}\text { Freitas et } \\
\text { al., } 2015\end{array}$ & $\begin{array}{c}\text { Acta Paulista } \\
\text { de Enfermage } \\
\mathrm{m}\end{array}$ & $\begin{array}{l}\text { Oeste de } \\
\text { Minas } \\
\text { Gerais }\end{array}$ & $\begin{array}{l}\text { Descritivo, } \\
\text { Transversal e } \\
\text { Analítico }\end{array}$ & $\begin{array}{l}\text { Analisar } \\
\text { fatores } \\
\text { associados à } \\
\text { síndrome } \\
\text { metabólica e à } \\
\text { qualidade de vida } \\
\text { em } \\
\text { pacientes com } \\
\text { esquizofrenia } \\
\text { refratária em uso } \\
\text { de clozapina }\end{array}$ \\
\hline $\begin{array}{l}\text { Sampaio } \\
\text { et al., } \\
2016\end{array}$ & $\begin{array}{l}\text { Revista } \\
\text { Brasileira em } \\
\text { Promoção da } \\
\text { Saúde }\end{array}$ & Fortaleza & $\begin{array}{c}\text { Transversal } \\
\text { descritivo }\end{array}$ & $\begin{array}{l}\text { Caracterizar } \\
\text { perfil } \\
\text { socioeconômico, } \\
\text { nutricional e os } \\
\text { fatores de risco } \\
\text { cardiometabólico } \\
\text { de pacientes com } \\
\text { esquizofrenia em } \\
\text { uso } \\
\text { antipsicóticos de } \\
\text { atendidos em um } \\
\text { hospital público } \\
\text { de Fortaleza/CE. }\end{array}$ \\
\hline
\end{tabular}


Quadro 3. Principais diagnósticos psíquicos encontrados nos artigos

\begin{tabular}{|l|c|}
\hline \multicolumn{1}{|c|}{ Autores } & Transtorno Psiquiátrico \\
\hline Seow et al., 2017; Jeon et al., 2017; Alswalt & Esquizofrenia $(n=13)$ \\
et al., 2017; Paredes et al., 2014; Saloojee et & \\
al., 2014; Silva et al., 2019; Olmos et al., & \\
2017; Freitas et al., 2016; Santini et al., 2016; & \\
Saloojee et al., 2016; Sugawara et al., 2014; & \\
Freitas, 2015; Sampaio et al., 2016 & \\
\hline Santini et al, 2016 & Transtorno Depressivo Maior $(n=2)$ \\
\hline Santini et al, 2016 & Transtorno do Humor Bipolar $(n=2)$ \\
\hline
\end{tabular}

Quanto à utilização de antipsicóticos constatou-se predominância na utilização de antipsicóticos atípicos em uma considerável parcela dos estudos selecionados para esta revisão $(n=10)$ (JEON et al., 2017; PAREDES et al., 2014; SALOOJEE et al., 2014; SILVA et al., 2019; FREITAS et al., 2016; SANTINI et al., 2016; OLMOS et al., 2017; SALOOJE et al., 2016; FREITAS et al., 2015; SAMPAIO et al., 2016) seguidos da utilização de antipsicóticos de primeira geração ( $n=2)$ (SEOW et al., 2017; ALSWALT et al., 2017), sendo que um número menor se encontrava em uso de ambas as classes.

Além disso, identificou-se um artigo que não referiu as classes de antipsicóticos em seu estudo (SUGAWARA et al., 2014), uma vez que o mesmo focou em analisar a abordagem dos psiquiatras no rastreamento, monitoramento e prevenção dos riscos metabólicos em usuários de antipsicóticos, o qual foi incluído para análise devido sua relevância de contribuição para o presente estudo (Quadro 4).

De acordo com Olmos et al (2017) e Paredes et al (2014) no que tange ao tratamento medicamentoso, os antipsicóticos de segunda geração representam os fármacos mais comumente empregados no tratamento de pacientes diagnosticados com esquizofrenia.

Os antipsicóticos são divididos em duas classes terapêuticas que diferem um do outro, basicamente, por potência terapêutica e afinidade em relação aos receptores, de modo que tais afinidades estão diretamente relacionadas com a eficácia clínica, resposta terapêutica, bem como para o desenvolvimento de efeitos adversos (SILVA et al., 2019). 
Usualmente os antipsicóticos são classificados como antipsicóticos convencionais clássicos, conhecidos como de primeira geração ou "típicos" e antipsicóticos atípicos, denominados como de segunda geração, havendo evidências que tanto as classes típicas quanto atípicas estão associadas a alterações metabólicas. Sabe-se que a ação terapêutica dos antipsicóticos típicos está relacionada ao bloqueio nos receptores de dopamina, o que tem sido associado ao aparecimento de efeitos colaterais, dentre os quais a sonolência e ganho de peso, que são resultados de bloqueio dos receptores histamínicos (SAMPAIO et al., 2016).

Os antipsicóticos atípicos representam uma classe que age de maneira a provocar menos efeitos extrapiramidais, proporcionando assim uma melhor adesão do paciente ao tratamento. Porém, os antipsicóticos de segunda geração não estão imunes de ocasionar sintomas adversos. A indução do ganho de peso promovidos por essa classe já era conhecida cientificamente. Porém, o aumento do risco de dislipidemias, diabetes, doenças cardiovasculares e morte prematura são itens que necessitam de mais estudos que busquem compreender essa associação. (SILVA et al., 2019; OLMOS et al., 2017).

\section{Quadro 4. Principais antipsicóticos utilizados}

\begin{tabular}{|l|c|}
\hline \multicolumn{1}{|c|}{ Autores } & Fármacos Psicotrópicos \\
\hline Seow et al., 2017; Alswalt et al., 2017 & Típicos $(\mathrm{n}=2)$ \\
\hline Jeon et al., 2017; Paredes et al., 2014; & Atípicos $(\mathrm{n}=10)$ \\
Saloojee et al., 2014; Silva et al., 2019; Freitas & \\
et al., 2016; Santini et al., 2016; Olmos et al., & \\
2017; Saloojee et al., 2016; Freitas et al., & \\
2015; Sampaio et al., 2016. & \\
\hline Sugawara et al., 2014 & Não referido $(\mathrm{n}=1)$ \\
\hline
\end{tabular}

No que diz respeito à identificação da Síndrome Metabólica, visualizou-se que quatro autores (SEOW et al., 2017; FREITAS et al., 2016; SANTINI et al., 2016; FREITAS et al., 2015) utilizaram os Critérios do National Cholesterol Education Program - Adult Treatment Panel III (NCEP ATP III) enquanto houve um predomínio de autores $(n=6)$ (JEON et al., 2016; SUGAWARA et al., 2014; 
SAMPAIO et al., 2016; SALOOJEE et al., 2014; OLMOS et al., 2017; PAREDES et al., 2014) que não utilizaram critérios para classificação da Síndrome Metabólica, analisando assim em seus estudos os riscos metabólicos por meio de fatores individuais em usuários de antipsicóticos.

Identificou-se em menor proporção (ALSWALT et al., 2017; SILVA et al., 2019; SALOOJEE et al., 2016) a utilização de distintos critérios para classificação da Síndrome Metabólica, sendo eles: International Diabetes Federation (IDF), the American Latin Association of Diabetes (ALAD), Joint Interim Statement (JIS).

A Síndrome Metabólica, por sua vez, caracteriza-se por associação de diferentes fatores, incluindo acúmulo considerável de gordura abdominal, alterações na glicemia, dislipidemias e alterações na pressão arterial sistêmica, condições clínicas que estão relacionadas ao aumento do risco cardiovascular. O estudo de Síndrome Metabólica em indivíduos diagnosticados com esquizofrenia, em particular, tem recebido atenção significativa na literatura, devido a maior morbidade e mortalidade observadas nesse grupo quando comparado com a população em geral (SEOW et al., 2017; JEON et al., 2017; ALSWAT et al., 2017).

A menor expectativa de vida verificada nos indivíduos diagnosticados com esquizofrenia pode ser explicada, dentre outros fatores, em decorrência do aumento de risco de Doenças Cardiovasculares (DCV) o que pode ocorrer em razão desses fatores de risco estarem presente nesta população, tais como obesidade, hiperglicemia, hipertensão, dislipidemias e tabagismo. Além disso, o estilo de vida e os fármacos antipsicóticos utilizados no tratamento podem contribuir para aumentar os fatores de risco de DCV (SANTINI et al., 2016).

Observou-se que houve prevalência de associação significativa entre antipsicóticos atípicos e Síndrome Metabólica (JEON et al., 2017; SILVA et al., 2019; OLMOS et al., 2017; SANTINI et al., 2016; FREITAS et al., 2015). Por outro lado, em menor proporção, constataram-se alterações metabólicas por análise de fatores individuais em indivíduos expostos à antipsicóticos, não havendo, assim, utilização de critérios para classificação da Síndrome Metabólica (PAREDES et al., 2014; SALOOJEE et al., 2014; SALOOJEE et al., 2016; SAMPAIO et al., 2016). 
Quadro 5. Critérios utilizados para diagnóstico da Síndrome Metabólica

\begin{tabular}{|l|c|}
\hline \multicolumn{1}{|c|}{ Autores } & $\begin{array}{c}\text { Critérios para Classificação } \\
\text { da SM }\end{array}$ \\
\hline $\begin{array}{l}\text { Seow et al., 2017; Freitas et al., 2016; } \\
\text { Santini et al., 2016; Freitas et al., 2015 }\end{array}$ & NCEP ATP III $(n=4)$ \\
\hline Alswalt et al., 2017 & IDF $(n=1)$ \\
\hline Silva et al., 2019 & ALAD $(n=1)$ \\
\hline Saloojee et al., 2016 & JIS $(n=1)$ \\
\hline $\begin{array}{l}\text { Jeon et al., 2016; Sugawara et al., 2014; } \\
\text { Sampaio et al., 2016; Saloojee et al., 2014; } \\
\text { Olmos et al., 2017; Paredes et al., 2014 }\end{array}$ \\
\hline
\end{tabular}

As razões para essa considerável prevalência ainda são muito controversas, principalmente em face da possível participação dos medicamentos antipsicóticos na gênese das anormalidades metabólicas. A esquizofrenia por si só já representa um fator de risco para as anormalidades que compõem a Síndrome Metabólica e, neste contexto, acredita-se que as medicações atípicas ou de segunda-geração possuem um papel importante na potencialização do risco (SILVA et al., 2019). A relação precisa entre antipsicóticos e o desenvolvimento de Síndrome Metabólica ainda permanece incerta, podendo-se afirmar com clareza que pessoas tratadas com esses medicamentos apresentam Síndrome Metabólica em uma frequência superior à da população geral (SANTINI et al., 2016).

Um dos obstáculos enfrentados no desenvolvimento de estudos relacionados à Síndrome Metabólica está associado à ausência de consenso quanto à sua definição, bem como nos pontos de corte de seus componentes, impactando tanto na prática clínica como nas políticas de saúde (FREITAS, 2015). Com isso, vale salientar que apesar de extensa investigação e discussão, ainda não existe um padrão-ouro para a definição de Síndrome Metabólica, que continua na pauta de vários debates e estudos (SEOW et al., 2017). 
Quadro 6. Prevalência de Síndrome Metabólica (SM) associada à classe Antipsicótica

\begin{tabular}{|c|c|}
\hline Autores & Prevalência da SM \\
\hline $\begin{array}{l}\text { Jeon et al., 2017; Silva et al., 2019; } \\
\text { Olmos et al., 2017; Santini et al., } \\
\text { 2016; Freitas et al., } 2015\end{array}$ & $\begin{array}{l}\text { Houve associação significativa entre } \\
\text { antipsicóticos atípicos e SM }(n=5)\end{array}$ \\
\hline Alswat et al., 2017 & $\begin{array}{l}\text { Houve associação significativa entre } \\
\text { SM e antipsicóticos típicos }(n=1)\end{array}$ \\
\hline $\begin{array}{l}\text { Paredes et al., 2014; Saloojee et al., } \\
2014 \text {; Saloojee et al., 2016; } \\
\text { Sampaio et al., } 2016\end{array}$ & $\begin{array}{l}\text { Não classificou a SM, porém houve } \\
\text { alterações metabólicas }(n=4)\end{array}$ \\
\hline Freitas et al., 2016 & $\begin{array}{l}\text { Houve associação significativa com } \\
\text { ambas classes de antipsicóticos }(n=1)\end{array}$ \\
\hline Seow et al., 2017 & $\begin{array}{l}\text { Não houve associação significativa } \\
(n=1)\end{array}$ \\
\hline
\end{tabular}

Deste modo, em estudos realizados por ausência de consenso quanto à sua definição em pacientes institucionalizados em hospitais de longa permanência em Singapura, que receberam assistência por um período mínimo de um ano, observou-se que houve aplicação de dois critérios distintos para definição de Síndrome Metabólica no mesmo grupo: quando aplicados os critérios de AHA/NHLBI verificou-se uma prevalência de $51,9 \%$ e de $26,9 \%$ quando avaliados pelos critérios de NCEP ATP III (SEOW et al., 2017).

Estudos realizados por Sugawara et al (2014) para identificar a prática de psiquiatras frente a riscos metabólicos em pacientes com esquizofrenia em uso de antipsicóticos, dentre 4.482 questionários respondidos, 85,2\% dos entrevistados demonstraram ciência quanto aos riscos metabólicos observados após o início do tratamento, porém um número limitado relatou viabilidade em realizar monitoramento a fim de reduzir os eventos adversos.

A pesquisa apresenta limitações no que diz respeito ao monitoramento e conhecimento do perfil metabólico dos usuários antes da utilização dos fármacos psicoativos para comparar com o perfil metabólico após a utilização dos medicamentos supracitados, bem como a definição de um padrão-ouro para classificar a Síndrome Metabólica, além da ausência de 
investigação do consumo alimentar e atividade física, visto que representam importantes fatores na busca por uma associação mais fidedigna.

\section{Conclusão}

Dado o exposto, observou-se nesta revisão que uma quantidade expressiva dos estudos $(n=10)$ relacionou associação significativa entre antipsicóticos atípicos e alterações metabólicas.

Diante das distintas classificações existentes, há um questionamento quanto à legitimidade das diversas junções dos critérios diagnósticos para Síndrome Metabólica, uma vez que pacientes com dois componentes da síndrome já apresentam risco aumentado, apesar de não preencherem todos os critérios diagnósticos, além de outras considerações em relação aos diferentes valores considerados normais. Deste modo, é interessante que exista um bom senso e análise de todo o contexto do indivíduo ao realizar estudos sobre Síndrome Metabólica.

Sugere-se que a investigação de hábitos alimentares e nível de atividade física seja realizada, visto que são fatores imprescindíveis na associação entre riscos metabólicos e uso de antipsicóticos. Outro ponto a ser considerado é o estímulo para o desenvolvimento de medidas que visem um estilo de vida mais saudável, autonomia e garantia de qualidade de vida de indivíduos expostos a antipsicóticos.

Propõe-se então que além do cuidado clínico, psiquiátrico e psicológico comumente ofertados aos indivíduos com transtornos mentais, haja promoção de um acompanhamento nutricional desses pacientes, buscando empregar intervenções nutricionais satisfatórias que objetivem tratar o sobrepeso e obesidade comumente observados nos mesmos, visando o desenvolvimento de estratégias que possibilitem a redução de riscos metabólicos e doenças crônicas não-transmissíveis existentes na população.

Dessa forma, apesar de vários achados científicos correlacionarem a utilização de antipsicóticos com alterações metabólicas em pacientes com transtornos mentais, esta revisão tenciona contribuir de forma importante para a literatura, tendo em vista que os resultados obtidos representam um ponto de partida para o desenvolvimento de pesquisas que busquem o planejamento e 
implantação de intervenções que visem à minimização e resolução da problemática encontrada.

\section{Referências}

ALSWAT, K. A. et al. Prevalence of Metabolic Syndrome in the Hospitalized Psychiatric Patients. Medical Archive, v. 71, n. 6, p. 412-416, 2017.

FREITAS, P. H. B. Metabolic syndrome prevalence in patients with refractory schizophrenia. Ciência y Enfermeria XXII, v. 3, p. 11-24, 2016.

FREITAS, P. H. B. Refractory schizophrenia: quality of life and associated factors. Acta Paulista de Enfermagem, v. 29, n. 1, p. 60-8, 2015.

JEON, S. W. et al. Unresolved Issues for Utilization of Atypical Antipsychotics in Schizophrenia: Antipsychotic Polypharmacy and Metabolic Syndrome. International Journal of Molecular Sciences. v. 18, n. 2174, 2017.

OLMOS, I. et al. Correlación de las concentraciones plasmáticas de clozapina con el síndrome metabólico. Revista de Psiquiatría del Uruguay, v. 81, n. 2, p. 101-105, 2017.

PAREDES, R. M. et al. Metabolomic profiling of schizophrenia patients at risk for metabolic syndrome. International Journal of Neuropsychopharmacology, v. 17, p. 1139-1148, 2014.

ROCHA, B. S., et al. Psicofármacos na Estratégia Saúde da Família: perfil de utilização, acesso e estratégias para a promoção do uso racional. Ciência Saúde Coletiva, Rio de Janeiro, v. 18, n. 1, p. 3291-3300, nov. 2013.

SALOOJEE, S. et al. Metabolic Syndrome in South African Patients with Severe Mental Illness: Prevalence and Associated Risk Factors. Plos One, v. 16, 2016.

SALOOJEE, S. et al. Very low rates of screening for metabolic syndrome among patients with severe mental ilnness in Durban, South Africa. BMC Psychiatry, v. 14, n. 228, 2014.

SAMPAIO, L. R. L. Socioeconomic and nutritional profile and cardiometabolic risk factors of schizophrenic patients treated with antipsychotics: a reflection for nutritional intervention. Revista Brasileira em Promoção da Saúde, Fortaleza, 29(1): 60-67, jan./mar., 2016.

SANTINI, I. et al. The metabolic syndrome in an Italian psychiatric sample: a retrospective chart review of inpatients treated with antipsychotics. Rivista di Psichiatria. v. 51, n. 1, p. 37-42, 2016.

SEOW, L. S. E. et al. Metabolic syndrome and cardiovascular risk among institutionalized patients with schizophrenia receiving long term tertiary care. Comprehensive Psychiaty, v. 74, p. 196 - 203, 2017. 
SILVA, D. G. C. et al. Schizophrenia: effects of aripiprazole in metabolic syndrome. Brazilian Journal of Pharmaceutical Sciences, v. 55, n. 7840, 2019.

SUGAWARA, N. et al. Psychiatrists' Attitudes toward Metabolic Adverse Events in Patients with Schizophrenia. Plos One, v. 9, n. 1, 2014.

XAVIER, M. S. et al. O significado da utilização de psicofármacos para indivíduos com transtorno mental em acompanhamento ambulatorial. Escola Anna Nery Revista de Enfermagem, Santa Maria, v. 18, n. 2, p. 323-329, abr./jun. 2014.

ZORTEA, K. et al. Estado nutricional de pacientes com esquizofrenia frequentadores do Centro de Atenção Psicossocial (CAPS) do Hospital de Clínicas de Porto Alegre. Jornal Brasileiro de Psiquiatria, Rio de Janeiro, vol. 59, n. 2, p. 126-130, 2010. 